\title{
Genetic Characterization of Campylobacter Jejuni and C. coli Isolated From Broilers Using flaA PCR-Restriction Fragment Length Polymorphism Method in Shiraz, Southern Iran
}

\author{
Rahem Khoshbakht ${ }^{1, *}$; Mohammad Tabatabaei ${ }^{2}$; Saeid Hosseinzadeh ${ }^{3}$; Hesamaddin \\ Shirzad Aski ${ }^{2}$; Saeed Seifi ${ }^{1}$ \\ ${ }^{1}$ Department of Food Hygiene, Faculty of Veterinary Medicine, Amol University of Special Modern Technologies, Amol, IR Iran \\ ${ }_{2}^{2}$ Department of Pathobiology, School of Veterinary Medicine, Shiraz University, Shiraz, IR Iran \\ ${ }^{3}$ Department of Public Health and Food Hygiene, School of Veterinary Medicine, Shiraz University, Shiraz, IR Iran \\ *Corresponding author: Rahem Khoshbakht, Department of Food Hygiene, Faculty of Veterinary Medicine, Amol University of Special Modern Technologies, Amol, IR Iran. Tel: \\ + 98 - 911919059, Fax: + 98 -1212271054, E-mail: Khoshbakht.r@gmail.com
}

Received: February 25, 2014; Revised: June 13, 2014; Accepted: July 21, 2014

\begin{abstract}
Background:Thermophilic campylobacters, particularly Campylobacterjejuni and C.coliarethemainagents of humancampylobacteriosis. Campylobacter contaminated chicken products is the most important source of foodborne gastroenteritis. Evaluation of genetic diversity among Campylobacter population is critical for understanding the epidemiology of this bacterium and developing effective control strategies against Campylobacter infections and other related disorders.

Objectives: The aim of this study was to investigate the polymorphism of thermophilic Campylobacter isolated from broiler fecal samples in Shiraz, southern Iran.

Materials and Methods: Ninety Campylobacter isolates were recovered from broiler feces using enrichment process followed by cultivation method. The isolates were species typing on the basis of polymerase chain reaction (PCR) detection of 16SrRNA and multiplex PCR for determining two thermophilic species. To evaluate strain diversity of thermophilic Campylobacter isolates, flaA PCR-Restriction Fragment Length Polymorphism (RFLP) was performed using DdeI restriction enzyme.

Results: All 90 Campylobacter isolates confirmed by m-PCR were successfully typed using flaA-PCR-RFLP. Eleven different types were defined according to flaA-typing method and the RFLP patterns were located at three separate clusters in RFLP image analysis dendrogram.

Conclusions: Campylobacterjejuni isolates significantly showed more variety than C. coli isolates. A relatively low genetic diversity existed among C. jejuni and C. coli isolated from broilers in Shiraz, southern Iran. In our knowledge, this was the first report of genetic diversity among broiler originated human pathogen thermophilic campylobacters in Shiraz, southern Iran.
\end{abstract}

Keywords: Campylobacter Jejuni; C. coli; Restriction Fragment Length Polymorphism; Iran

\section{Backgrounds}

Thermophilic Campylobacter species, particularly Campylobacter jejuni and C. coli are zoonotic bacteria frequently associated with human diarrhea in developing and industrialized countries (1). Campylobacter infections are acquired by ingestion of contaminated food, water, or milk, but the sources and transmission routes of campylobacteriosis in humans remain debatable. Numerous vehicles for transmission of Campylobacter infection have been described; however, chicken appears to be the most significant agent for sporadic cases (2-4). To reduce the occurrence of campylobacteriosis in humans, it is essential to improve general understanding of the epidemiology of infection.

The use of conventional bacteriological tests for differentiation and species identification of campylobacters is often hampered by the fact that these bacteria are fastidi- ous and possess few distinguishing biochemical characteristics (5). In the past decades, various methods have been developed for discrimination of Campylobacter at the DNA levels to trace various sources of infection and genotyping replaced the traditional typing methods such as serotype classifications (6-8). Among various genotypic methods, PCR-Restriction Fragment Length Polymorphism analysis of the flagellin-A gene (flaA) is widely used because of its rapidity, ease and cost-effectiveness (9). The existence of extremely conserved and inconsistent regions in flaA gene makes the locus proper for scrutiny of the effects of different restriction enzymes (10).

\section{Objectives}

The aim of this study was to determine genetic diversity

Copyright (C) 2015, Ahvaz Jundishapur University of Medical Sciences. This is an open-access article distributed under the terms of the Creative Commons Attribution-NonCommercial 4.0 International License (http://creativecommons.org/licenses/by-nc/4.0/) which permits copy and redistribute the material just in noncommercial usages, provided the original work is properly cited. 
of random collection of C. jejuni and C. coli strains isolated from broiler feces in Shiraz, southern Iran, using PCRRFLP and investigate the association of Campylobacter species and genetic polymorphism.

\section{Materials and Methods}

Totally, 42 C. jejuni and 48 C. coli were recovered from fecal samples of the broilers in 10 visits of sampling from Shiraz slaughterhouse in January 2012. The fecal samples were collected in Tryptic Soy Broth (TSB) broth tubes by sterile gloves and brought to the laboratory on ice packs in less than six hours. To eliminate other bacteria, $0.8 \mu \mathrm{m}$ membrane filter was used and $250 \mu \mathrm{L}$ of filtered samples was cultured in an enriched broth media [TSB (30 g/L), dextrose (2.5 g/L), sodium thioglycolate (0.5 g/L), Rifampicin (10 mg/L), Trimethoprim (10 mg/L), Vancomycin (10 mg/L), Ceftriaxone (10 mg/L), Amphotericin -B (10 mg/L)], incubated in a microaerophilic atmosphere (Merck, Germany) at $37^{\circ} \mathrm{C}$ for 4 hours, followed by incubation at $42^{\circ} \mathrm{C}$ for 44 hours. Thereafter, $50 \mu \mathrm{L}$ of enriched samples in TSB was cultured on selective agar [brucella agar base $(41 \mathrm{~g} / \mathrm{L})$, and above antibiotics with identical dose] (11). All culture media were from Merck, Germany. The growth of thermophilic campylobacters was detected by their typical appearance on culture media, i.e. the presence of flat grayish colonies like droplets of water sprayed on the medium. Preliminary identification of Campylobacter species was based on phenotypic characteristics such as colony appearance, Gram staining, microscopic morphology, oxidase and catalase reaction, fermentation of glucose and nitrate reduction (2). The type strains Campylobacter jejuni (ATCC 33291) and Campylobacter coli (RTCC 2541) were included as positive controls for culture identification of isolates.

\subsection{DNA Extraction}

DNA extraction was performed using the phenol-chloroform extraction technique previously described by Khoshbakht et al. (12). Briefly, a loopful colony of each isolates on agar plate was picked and suspended in 200 $\mu \mathrm{L}$ distilled water. After vortexing, samples were centrifuged at $10000 \times \mathrm{g}$, the supernatants were discarded before adding $250 \mu \mathrm{L}$ of buffer 1 (resuspension solution contained $100 \mu \mathrm{g} / \mathrm{mL}$ RNase) and $250 \mu \mathrm{L}$ buffer 2 (Lysis buffer solution contained Tris-HCl and EDTA), a $550 \mu \mathrm{L}$ saturated phenol was then added, mixed thoroughly and centrifuged at $8000 \times \mathrm{g}$. The supernatant was collected into a new tube; $550 \mu \mathrm{L}$ of the phenol was then added and centrifuged at $8000 \times \mathrm{g}$ again. The supernatant aqueous phase was collected into a fresh tube, previous to adding sodium acetate $(2 \mathrm{M}, \mathrm{pH}$ 5.2, $0.1 \times$ volume of each aliquot). Then $1.5 \mathrm{~mL} 100 \%$ ethanol were added to the aliquots and mixed, kept at $-20^{\circ} \mathrm{C}$ for 1 hour, centrifuged at 12,000 $\times \mathrm{g}$, the supernatant was then discarded and DNA pellet was washed by $80 \%$ ethanol, before being dried and suspended in $30 \mu \mathrm{L}$ of $1 \times$ TE buffer until use.

\subsection{Simple and Multiplex PCR Assay}

Simple and multiplex PCR reactions were performed for identification of Campylobacter genus, C. jejuni and C. coli species, respectively. PCR amplifications were performed in a final volume of $25 \mu \mathrm{L}$. The reaction mixtures consisted of $2 \mu \mathrm{L}$ DNA template, $2.5 \mu \mathrm{L} 10 \times$ PCR buffer [ 75 mM Tris$\mathrm{HCl}, \mathrm{pH}$ 9.0, $2 \mathrm{mM} \mathrm{MgCl}$, $\left.50 \mathrm{mM} \mathrm{KCl}, 20 \mathrm{mM}\left(\mathrm{NH}_{4}\right)_{2} \mathrm{SO}_{4}\right]$, (CinnaGen, Iran), $1 \mu \mathrm{L}$ dNTPs $(50 \mu \mathrm{M})$, (CinnaGen, Iran), $1 \mu \mathrm{L}$ (1U Ampli Taq DNA polymerase), (CinnaGen, Iran), 1 $\mu \mathrm{L}(25 \mathrm{pmol})$ from the forward and reverse primers (CinnaGen, Iran), shown in Table 1 and the volumes of the reaction mixtures were reached to $25 \mu \mathrm{L}$ using distilled deionized water. The thermal cycler (MJ mini, BioRad, USA) was adjusted under the following conditions: initial denaturation at $94^{\circ} \mathrm{C}$ for 5 minutes, followed by 35 cycles of denaturation at $94^{\circ} \mathrm{C}$ for 1 minute, annealing as shown in Table 1 for 1 minute and extension at $72^{\circ} \mathrm{C}$ for 1 minute. Final extension was performed at $72^{\circ} \mathrm{C}$ for 10 minute and the PCR products were remained in the thermal cycler at $4^{\circ} \mathrm{C}$ until they were collected. Amplified products were separated by electrophoresis in 1.5\% agarose gel stained with ethidium bromide. 100 bp DNA ladder was used as molecular size markers. Visualization was undertaken using a UV transilluminator (BTS-20, Japan). The type strains C. jejuni (ATCC 33291) and C. coli (RTCC 2541) were included as positive controls for PCR identification of the isolates and the master mix without sample DNA used as negative control.

\subsection{FlaA PCR-Restriction Fragment Length Poly- morphism}

Isolates identified as $C$. jejuni and $C$. coli by m-PCR were typed by PCR-RFLP for the flaA gene. A fragment of $1725 \mathrm{bp}$ (Table 1 ) of the flaA gene was amplified in a PCR reaction using a pair of specific primers listed in Table 1 previously described by Nachamkin et al. (13). Seven microliters of all the amplicons were restricted with $4 \mathrm{U}$ DdeI (Thermo scientific, Germany) in $1.5 \mu \mathrm{L} 10 \times$ recommended restriction buffer in the final volume of $20 \mu \mathrm{L}$ and then were incubated at $37^{\circ} \mathrm{C}$ for 15 hours. The digested PCR products $(15 \mu \mathrm{L})$ were immediately separated on $2.5 \%$ agarose gel stained with ethidium bromide. Bands were photographed under UV transilluminator and the results were evaluated manually. The 50 bp DNA ladder (CinnaGen, Iran) was used as molecular marker to estimate the size of bands. Dendrogram was constructed based on the obtained DNA fragment patterns using Phoretix 1D Version 10 software.

\subsection{Statistical Analysis}

Statistical analysis was performed using SPSS version 12.0.1. Discrete variables were expressed as percentages and proportions were compared using Chi-squared test with the significance level defined at $\mathrm{P}<0.05$. 
Table 1. Nucleotide Sequences used as Primers in the PCR Reaction for Identification of Campylobacter Genus and C. Jejuni and C. Coli Species ${ }^{\text {a }}$

\begin{tabular}{|c|c|c|c|c|c|}
\hline Name of Primer & Sequence $\left(5^{\prime}-3^{\prime}\right)$ & Target Gene & Annealing Temperature, ${ }^{\circ} \mathrm{C}$ & Product Size, bp & Reference \\
\hline MapA & & $\operatorname{mapA}($ (C. jejuni) & 52 & 589 & (14) \\
\hline $\mathrm{F}$ & СТATTTTATTTTTGAGTGCTTGTG & & & & \\
\hline $\mathrm{R}$ & GCTTTATTTGCCATTTGTTTTATTA & & & & \\
\hline Coli & & ceuE (C. coli) & 52 & 462 & (14) \\
\hline F & ААТTGAАAATTGCTCСАAСТАТG & & & & \\
\hline $\mathrm{R}$ & TGATTTTATTATTTGTAGCAGCG & & & & \\
\hline PLO6 & GGTTAAGTCCCGCAACGAGCCGC & $\begin{array}{l}\text { 16SrRNA (genus } \\
\text { specific) }\end{array}$ & 50 & 283 & (15) \\
\hline CAMPC5 & GGCTGATCTACGATTACTAGCGAT & $\begin{array}{l}\text { 16SrRNA (genus } \\
\text { specific) }\end{array}$ & 50 & 283 & (15) \\
\hline Fla & & flaA (RFLP) & 45 & 1725 & (13) \\
\hline 1 & GGATITCGTATTAACACAAATGGTGC & & & & \\
\hline 2 & СTGTAGTAATCTTAAAACATTTG & & & & \\
\hline
\end{tabular}

a Abbreviations: F, forward; R, reverse; RFLP, restriction fragment length polymorphism.

Table 2. flaA Typing Results of C. Jejuni and C. Coli Isolates Recovered From Broiler Fecal Samples a

\begin{tabular}{lcccccccccccc}
\hline \multirow{2}{*}{ Species } & No. & \multicolumn{10}{c}{ Occurrence of Different flaA-RFLP Patterns } \\
\cline { 2 - 13 } & & F1 & F2 & F3 & F4 & F5 & F6 & F7 & F8 & F9 & F10 & F11 \\
\hline C. jejuni & 42 & $8(19)$ & $4(9.5)$ & $49.5)$ & $0(0)$ & $3(7.1)$ & $2(4.7)$ & $7(16.6)$ & $2(4.7)$ & $4(9.5)$ & $6(14.2)$ & $2(4.7)$ \\
C. coli & 48 & $3(6.2)$ & $0(0)$ & $20(41.6)$ & $3(6.2)$ & $2(4.1)$ & $12(25)$ & $0(0)$ & $6(12.5)$ & $0(0)$ & $2(4.1)$ & $0(0)$ \\
Total & 90 & $11(12.2)$ & $4(4.4)$ & $24(26.6)$ & $3(3.3)$ & $5(5.5)$ & $14(15.5)$ & $7(7.7)$ & $8(8.8)$ & $4(4.4)$ & $8(8.8)$ & $2(2.2)$ \\
\hline
\end{tabular}

${ }^{\mathrm{a}}$ Data are presented as No. (\%)

\section{Results}

In total, 90 Campylobacter isolates including $48 \mathrm{C}$. coli and 42 C. jejuni were recovered from fecal samples and identified based on simple and multiplex PCR reactions. All 90 isolates were typed successfully and 11 different genotypes (Table 2) were defined by flaA-PCR-RFLP method. The most prevalent fla-typing pattern of the isolates was F3 (26.6\%). Among 42 C. jejuni originating from broiler fecal samples, 10 different types (all types except F4) were defined (Table 2 ) and the most common type was F1 (19\%). Among 48 C. coli isolates, seven types (F1, F3, F4, F5, F6, F8 and F10) were defined and the most common type was type F3 (41.6\%). Between 11 different types, four patterns (F2, F7, F9 and F11) were specific for C. jejuni and one pattern (F4) was specific for C. coli isolates. Statistical analysis of flaA-typing data showed that RFLP patterns F2 and F10 were significantly $(\mathrm{P}<0.05)$ predominant among C. jejuni and RFLP patterns F3 and F6 were significantly ( $\mathrm{P}$ $<0.05$ ) predominant among $C$. coli isolates. The phylogenetic analysis based on the dendrogram generated from RFLP-PCR demonstrated that $C$. jejuni and C. coli isolates belong to three clusters (I, II and III in Figure 1). 45.5\% (41/90) of isolates were found in cluster I and $27.7 \%$ (25/90) and $26.6 \%(24 / 90)$ of isolates belonged to clusters II and III, respectively. The dominant cluster of C. jejuni and

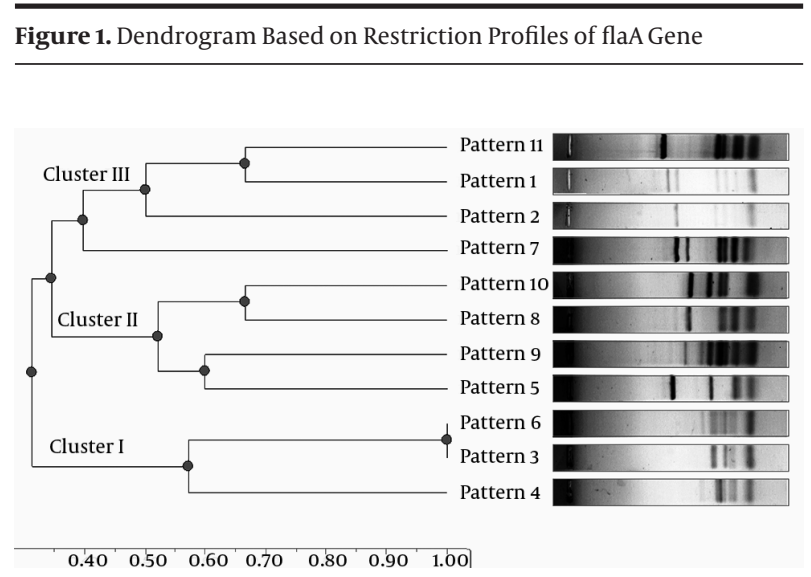

Unweighted Pair Group Method with Arithmetic Mean (UPGMA) with a dice coefficient distance tree was generated with Phoretix 1D Version 10 software. The numbers ( 0.40 to 1.00$)$ show the similarity scale. Circles define the point of the similarity level.

C. coli isolates was significantly $(\mathrm{P}<0.05)$ difficult, so that $72.9 \%$ (35/48) of C. coli belonged to cluster I, while 50\% (21/42) and 35.7\% (15/42) of C. jejuni belonged to clusters III and II, respectively (Figure 1 ). 


\section{Discussion}

Typing of Campylobacter isolates from different sources provides epidemiological information needed for infection control and contributes to risk evaluation of transmission. Genotype-based identification methods have been developed to avoid the problems caused by biochemical inertness of Campylobacter species. These methods are now more frequently used, but have yet to be standardized. The application of molecular typing methods can offer a constant and highly discriminatory investigation of bacterial isolates. The present study considered the heterogeneity among C. jejuni and C. coli isolates obtained from broiler feces by molecular methods for the first time in Iran.

PCR-RFLP genotyping method was used to explain genetic diversity among these isolates. All 90 isolates confirmed based on m-PCR were typed successfully using fla typing method. Harrington et al. (16) in their study compared three different methods of flaA-RFLP technique and showed that, full flaA gene and DdeI digestion are proper for fla-typing of campylobacters. Other similar studies indicated the benefits of full length flaA gene and DdeI $(17,18)$, which we used in this study. In the current study, 11 distinct flaA-types were obtained, using DdeI, which did not show high diversity among isolates compared with other studies on Campylobacter isolates from some other sources (19-22). These differences in genetic variation among thermophilic Campylobacter strains can be explained by geographical diversity, different number of isolates compared with other studies and difference in origin. Compared with other poultry-originated Campylobacter isolates in some studies (9), the isolates in the present study showed lower diversity, but some other investigations showed lower diversity (1).

Results showed that among 11 RFLP patterns, six (54.5\%) patterns were similar between C. jejuni and C. coli strains. Ertas et al. (1) showed seven different fla-types among $C$. jejuni and $C$. coli isolates, which all were similar among two species. Nevertheless, Oporto et al. (22) in analysis of thermophilic campylobacters from cattle, sheep and swine farms obtained 46 different patterns, which only five $(10.8 \%)$ were similar between $C$. jejuni and C. coli isolates. These data indicate low genetic diversity among our study broiler originated thermophilic campylobacters. More studies are necessary to evaluate genetic diversity differences between two thermophilic species. Isolation of Campylobacter from each cloacal swab, without resorting to pooling, made it possible to perform an epidemiological study using a molecular method such as flaA-typing to evaluate the relatedness of Campylobacter isolates of each farm.

Our isolates were not recovered from a single commercially reared flock and samples were collected randomly from separate flocks in slaughtering line at the end of the rearing period. Genetic differences between poultry-originated isolates may be due to variety in age of chickens, site of sampling and category of rearing (23). According to the results, C. jejuni isolates showed significantly $(\mathrm{P}<0.05)$ higher diversity (10 patterns) than $C$. coli isolates ( 7 patterns). In addition, predominant flaA-typing patterns of $C$. jejuni strains were significantly $(\mathrm{P}<0.05)$ different from $C$. coli isolates. Acik and Cetinkaya (21) showed that a mong 209 isolates, 28 different flaA types were found. Twenty-three flaA types were identified among 179 C. jejuni isolates and the remaining five from $C$. coli isolates. Using RFLP image analysis, dendrogram was drawn and we examined the genomic association between the Campylobacter isolates tested in the study. C.jejuni and C. coli were randomly distributed in three clusters, but most isolates were found in cluster I (Figure 1). The results showed that $C$. jejuni and $C$. coli isolates significantly $(\mathrm{P}<0.05)$ belonged to different clusters in phylogenic dendrogram drawn according to flaA-RFLP patterns. Clusters I and III had significant $(\mathrm{P}<0.05)$ relatedness with $C$. coli and $C$. jejuni, respectively. This can show the differences between fla-typing features of these two species, which can be observed by sequencing and comparison of all flaA PCR products. Three of four patterns, which were not present in $C$. coli isolates, were in cluster III.

In conclusion, the presence of campylobacters in broiler feces can contaminate the environmental and human food chain. Therefore, detection of Campylobacter spp. in broiler originated samples is important to identify possible sources of infection and to have a better understanding of the epidemiology of infection subtyping of isolates is considered essential. Results indicated that Flatyping is a simple and low-cost typing method characterized by a high level of discrimination power and it is an efficient tool for identifying any outbreak-related strains in short-term investigations. The results of this study revealed a comparatively low heterogeneity among C.jejuni and C. coli isolated from the broilers in southern Iran. In addition, results showed that the predominant RFLP patterns of $C$. coli and C. jejuni isolates can be significantly different and belong to various distinct phylogenic clusters. These molecular epidemiologic findings should be taken into account in the investigations towards developing effective control strategies against $C$. jejuni and C. coli infections.

\section{Acknowledgements}

The authors wish to thank the study participants for their contribution to the research, as well as current and past investigators and staff. The authors would specifically like to thank Amin Shahed for Laboratory assistance. This work was supported by School of Veterinary Medicine, Shiraz University, Iran.

\section{Authors' Contributions}

All Authors had equal contribution in the manuscript. 


\section{References}

1. Ertas HB, Cetinkaya B, Muz A, Ongor H. Genotyping of broileroriginated Campylobacter jejuni and Campylobacter coli isolates using fla typing and random amplified polymorphic DNA methods. Int J Food Microbiol. 2004;94(2):203-9.

2. Stern NJ, Fedorka-Cray P, Bailey JS, Cox NA, Craven SE, Hiett KL, et al. Distribution of Campylobacter spp. in selected U.S. poultry production and processing operations. J Food Prot. 2001;64(11):1705-10.

3. Stoyanchev T, Vashin I, Ring C, Atanassova V. Prevalence of Campylobacter spp. in poultry and poultry products for sale on the Bulgarian retail market. Antonie Van Leeuwenhoek. 2007;92(3):285-8.

4. Mdegela RH, Laurence K, Jacob P, Nonga HE. Occurrences of thermophilic Campylobacter in pigs slaughtered at Morogoro slaughter slabs, Tanzania. Trop Anim Health Prod. 2011;43(1):83-7.

5. Engvall EO, Brandstrom B, Gunnarsson A, Morner T, Wahlstrom $\mathrm{H}$, Fermer C. Validation of a polymerase chain reaction/restriction enzyme analysis method for species identification of thermophilic campylobacters isolated from domestic and wild animals. J Appl Microbiol. 2002;92(1):47-54.

6. Wassenaar TM, Newell DG. Genotyping of Campylobacter spp. Appl Environ Microbiol. 2000;66(1):1-9.

7. Caner V, Cokal Y, Cetin C, Sen A, Karagenc N. The detection of hipO gene by real-time PCR in thermophilic Campylobacter spp. with very weak and negative reaction of hippurate hydrolysis. Antonie Van Leeuwenhoek. 2008;94(4):527-32.

8. Findik A, Ica T, Onuk EE, Percin D, Kevenk TO, Ciftci A. Molecular typing and cdt genes prevalence of Campylobacter jejuni isolates from various sources. Trop Anim Health Prod. 2011;43(3):711-9.

9. Corcoran D, Quinn T, Cotter L, Whyte P, Fanning S. Antimicrobial resistance profiling and fla-typing of Irish thermophillic Campylobacter spp. of human and poultry origin. Lett Appl Microbiol. 2006;43(5):560-5.

10. Shi F, Chen YY, Wassenaar TM, Woods WH, Coloe PJ, Fry BN. Development and application of a new scheme for typing Campylobacter jejuni and Campylobacter coli by PCR-based restriction fragment length polymorphism analysis. J Clin Microbiol. 2002;40(5):1791-7.

11. Ansari-Lari M, Hosseinzadeh S, Shekarforoush SS, Abdollahi M, Berizi E. Prevalence and risk factors associated with campylobacter infections in broiler flocks in Shiraz, southern Iran. Int J Food Microbiol. 2011;144(3):475-9.

12. Khoshbakht R, Tabatabaei M, Hosseinzadeh S, Shekarforoush SS, Aski HS. Distribution of nine virulence-associated genes in Campylobacter jejuni and C. coli isolated from broiler feces in Shiraz,
Southern Iran. Foodborne Pathog Dis. 2013;10(9):764-70.

13. Nachamkin I, Bohachick K, Patton CM. Flagellin gene typing of Campylobacter jejuni by restriction fragment length polymorphism analysis. J Clin Microbiol.1993;31(6):1531-6.

14. Denis M, Soumet C, Rivoal K, Ermel G, Blivet D, Salvat G, et al. Development of a m-PCR assay for simultaneous identification of Campylobacter jejuni and C. coli. Lett Appl Microbiol. 1999;29(6):406-10.

15. Cardarelli-Leite P, Blom K, Patton CM, Nicholson MA, Steigerwalt AG, Hunter SB, et al. Rapid identification of Campylobacter species by restriction fragment length polymorphism analysis of a PCR-amplified fragment of the gene coding for $16 \mathrm{~S}$ rRNA.J Clin Microbiol.1996;34(1):62-7.

16. Harrington CS, Moran L, Ridley AM, Newell DG, Madden RH. Inter-laboratory evaluation of three flagellin PCR/RFLP methods for typing Campylobacter jejuni and C. coli: the CAMPYNET experience. J Appl Microbiol. 2003;95(6):1321-33.

17. Wieczorek K, Osek J. Identification of virulence genes in campylobacter jejuni and c. coli isolates by PCR. Bull Vet Inst Pulawy. 2008;52(1):211-6.

18. El-Adawy H, Hotzel H, Tomaso H, Neubauer H, Taboada EN, Ehricht $\mathrm{R}$, et al. Detection of genetic diversity in Campylobacter jejuni isolated from a commercial turkey flock using flaA typing, MLST analysis and microarray assay. PLoS One. 2013;8(2):e51582.

19. Fitzgerald C, Stanley K, Andrew S, Jones K. Use of pulsed-field gel electrophoresis and flagellin gene typing in identifying clonal groups of Campylobacter jejuni and Campylobacter coli in farm and clinical environments. Appl Environ Microbiol. 2001;67(4):1429-36.

20. Bang DD, Nielsen EM, Scheutz F, Pedersen K, Handberg K, Madsen M. PCR detection of seven virulence and toxin genes of Campylobacter jejuni and Campylobacter coli isolates from Danish pigs and cattle and cytolethal distending toxin production of the isolates. J Appl Microbiol. 2003;94(6):1003-14.

21. Acik MN, Cetinkaya B. The heterogeneity of Campylobacter jejuni and Campylobacter coli strains isolated from healthy cattle. Lett Appl Microbiol. 2005;41(5):397-403.

22. Oporto B, Esteban JI, Aduriz G, Juste RA, Hurtado A. Prevalence and strain diversity of thermophilic campylobacters in cattle, sheep and swine farms. J Appl Microbiol. 2007;103(4):977-84.

23. Datta S, Niwa H, Itoh K. Age-dependent variation of virulenceassociated genes retained in Campylobacter jejuni isolated from chickens in a poultry farm. J Vet Med Sci. 2009;71(9):1247-9. 